\title{
Research on Reconfigurable and Reliable Manipulators
}

\author{
(Report for Period February 15, 1992 - February 14, 1993)
}

\section{Project Investigators}

\author{
Professors Pradeep K. Khosla and Takeo Kanade
}

\author{
Address \\ Department of Electrical and Computer Engineering \\ and The Robotics Institute \\ Carnegie Mellon University \\ Pittsburgh, Pennsylvania 15213-3890
}

\section{DISCLAIMER}

\begin{abstract}
This report was prepared as an account of work sponsored by an agency of the United States This report was prepared as an account of work sponsored by agency thereof, nor any of their employees, makes any warranty, express or implied, or assumes any legal liability or responsibility for the accuracy, completeness, or usefulness of any information, apparatus, product, or process disclosed, or represents that its use would not infringe privately owned rights. Referprocess disclosed, or represents that its use wo specific commercial product, process, or service by trade name, trademark, manufacturer, or otherwise does not necessarily constitute or imply its endorsement, recommendation, or favoring by the United States Government or any agency thereof. The views and opinions of authors expressed herein do not necessarily state or reflect those of the United States Government or any agency thereof.
\end{abstract}

This is a yearly progress report for the above mentioned grant period. The current grant number is DOE-F902-89ER 14042. 


\section{Summary}

We have proposed the concept of reconfigurable manipulators as a solution to tasks that require varied configurations of manipulators. Using modules of varying sizes and performance specifications, it is possible to build manipulators to suit the task at hand. Our research is not only addressing the issue of creating such manipulators but also the interesting theoretical challenges posed in mapping tasks to manipulators. We have developed strategies that allow us to determine the kinematic and dynamic configuration of a nonredundant manipulator from task specifications such as reachability, obstacle avoidance, manipulability, joint, limits, and joint velocities and accelerations. We are presently extending this design methodology for design of redundant and fault tolerant manipulator systems. Further, we are also addressing issues in automatic generation of software for kinematics, dynamics, and conurollers for the configured manipulators. During the next year, we will be demonstrating automatic generation of software on the prototype system that we are building. We expect to have 4 joint and link modules available for this demonstration. The joint and link modules will also incorporate concepts for making power and data connections when the mechanical connection is made. Further, they will also include electronics, housed within the module, for communication and real-time control.

\section{Description of Our Work during the Period February 92 - February 93}

\subsection{Introduction}

The technology for robotic manipulators has been developed primarily for industrial applications where the task environment san be engineered or constrained. Consequently, the dexterity and versatility of today's robotic manipulators are far from sufficient to perform tasks in an unpredictable, less constrained, and changing environment. One of the important reasons for this has been the lack of research in manipulator designs that embody the principles of reconfigurability and modularity, and the corresponding theories that allow the user to automatically map the task requirements into a kinematic configuration and the corresponding controller requirements. A thorough survey of researchers in the area of robotics identified the importance and need for reconfigurability and modularity in manipulator designs in order to enhance their versatility.

Presently a specific manipulator is chosen for a specific task and if the task requirements were to change a different manipulator would be required. In dynamic environments that may be encountered in a nuclear facility or aboard a space station it is desirable to have a manipulator system that could, within reason, reconfigure itself and adapt to the new task requirements. For example, consider the use of manipulators in a field or in a nuclear facility that must assemble, and repair large and small structures (e.g. antenna towers, trusses) and equipment (e.g. vehicles, electronics). In such an environment, oftentimes widely varied 
. kinematic (e.g., workspace) and dynamic (e.g., speed, accuracy) performance is desired. Generally, either no single conventional manipulator is capable of delivering these requirements, or it is too expensive to prepare a large set of manipulators to accomplish these tasks. Moreover in some other situations, the manipulator kinematics and dynamics requirements may not be even known a priori, and yet a manipulator is to be deployed for performing the task. Thus, there exists a need for advanced manipulators that can be rapidly reconfigured to cover a wide range of task requirements.

To fulfill this need, we have been pursuing research, under a grant from DOE Office of Basic Energy Sciences for the period June 1989 - August 1992, on developing the theoretical basis and the technology for Reconfigurable Modular Manipulation System (RMMS). Unlike a conventional manipulator which has a fixed configuration, the RMMS consists of a set of interchangeable modules that can be rapidly assembled into a system of manipulators with appropriate configurations depending on the specific task requirement. For effective development and use of such a versatile and flexible system we have been pursuing a program of theoretical and experimental research aimed at developing the basis for next generation of autonomous manipulator systems. The RMMS concept extends the idea of autonomy from sensor-based to configuration based autonomy. One of the important components of our research is the development of design methodologies for mapping tasks into manipulator configurations and for automatic generation of manipulator specific algorithms (e.g., kinematics and dynamics) in order to make the hardware transparent to the user.

During this period of performance we have made significant progress towards accomplishing both the theoretical and experimental objectives of our grant. In the next section, we describe our accomplishments in further detail. In Section 1.2.1. we provide a detailed list of the publications and invention disclosures that have resulted from our research.

Our research has not only made contributions to the theory of reconfigurable and reliable (fault tolerant) manipulator systems but it has also impacted robotics in DOE. Based on our work, DOE has included modular and reconfigurable systems, in their 5 year plan for the ER\&WM (Environmental Restoration and Waste Management) program, as one of the key technologies that must be developed. In the following sections, we describe our accomplishments under the current grant.

\subsection{Accomplishments during the Current Grant Period}

In the following paragraphs we only provide a brief synopsis of our contributions during the current period. As an appendix, we have included publications that have resulted from this work and which provide more details.

Specifically, on the theoretical side, we have developed:

\section{Task Based Synthesis of Redundant and Non-Redundant manipulators}

Our past work (during the previous grant period June 89 - August 92) had developed methodologies that take the kinematic task characteristics as input and create 
a manipulator configuration (or Denavit-Hartenberg parameters of the manipulator) as output. This methodology was applicable only for non-redundant manipulators. Further, it was based on posing the entire design problem as a single optimization problem under the assumption that closed form inverse kinematics of an arbitrary non-redundant manipulator were available. While this assumption was reasonable for non-redundant manipulators, it posed several problems for extending this approach in a simple manner to the design of redundant manipulators. The main reason is that redundant manipulators are under constrained (they have infinite number of inverse kinematic solutions) and therefore require additional optimization criteria to constrain the solution.

During the current grant period, we have expanded our methodology to accomplish the design of redundant manipulators. We have taken the approach of formulating the problem as multiple optimization problems (based on genetic algorithms) that can be run in parallel. An obvious advantage of this approach is the reduction in time to realize an acceptable solution. More importantly, we have been able to relax the assumption that the inverse kinematics are known in closed form. We use genetic algorithms as the basis for our optimization. Using this approach, we have been able to demonstrate the design of a 7 degrees-of-freedom redundant manipulator for servicing the tiles of the space shuttle. Furthermore, we were able to develop algorithms that could synthesize both the kinematic configuration and the base location (of the manipulator) simultaneously. Design of base locations is important because in unstructured environments, it is not always possible to determine where the base of the rnanipulator must be placed to accomplish the task.

We have attached our paper based on this work as an appendix. This paper has been accepted for presentation and publication in the 1993 IEEE Conference on Robotics and Automation.

\section{Methodologies for design of Fault Tolerant Manipulators.}

During this reporting period, we have also been actively pursuing the development of design methodologies for fault tolerant systems. We define as fault tolerant manipulator as one that can effectively accomplish the task in the event of one or more simultaneous failures in the joints. An i-th order fault tolerant system can effectively tolerate $i$ joints that fail simultaneously. Due to the complexity of formulating the design of an -th order fault tolerant system, we have been concentrating on 1-st order fault tolerance.

As must be clear, a fault tolerant manipulator has to be redundant but a redundant manipulator is not necessarily fault tolerant. While our work on designing redundant manipulators is necessary to the solution of the fault tolerant design problem, it is not sufficient. While our preliminary results on addressing the design of fault tolerant manipulators have been very encouraging, a lot of work still remains to be done in this area. Using our methodology, we have been able to demonstrate that it is indeed possible to design spatial manipulators that are 1-st order fault tolerant. 
We have been able to show that at least 5 degrees-of-freedom are required for a simple positioning task. Presently, our methodology includes only the reachability condition for specifying a task. In order for such a design methodology to be useful, we must also include other criteria such as manipulability, obstacle avoidance, joint limits, etc. Our future work will address this issue.

We have attached our paper based on this work as an appendix. A shorter version of this paper was presented at the 1993 Allerton Conference held at Univ of Illinois, Urbana Champaign.

\subsubsection{MS/Ph.D. Thesis Supported under the Grant}

During this grant period, one student (Jin-Oh Kim) finished his Ph.D. thesis.

Christaan Pareedis, Presently Pursuing a Ph.D. degree, Expected Completion date for the Ph.D. is August 1995.)

Jin-Oh Kim, Task Based Design of Manipulators. Ph.D. thesis, Octcober, 1992. 

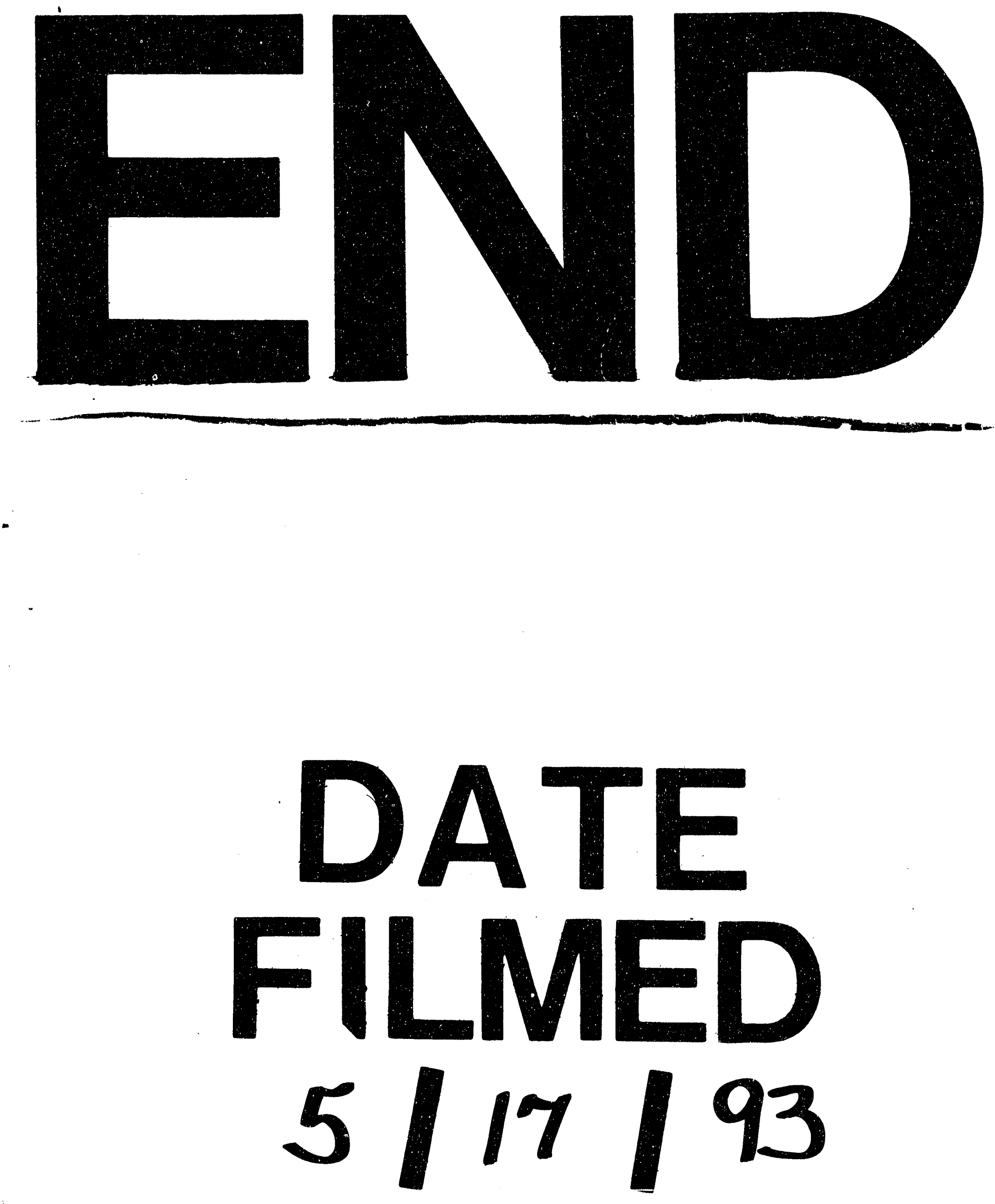
1 